\title{
ARTICLE
}

\section{Functional asymmetry of transmembrane segments in nicotinic acetylcholine receptors}

\author{
Jörg Grandl · Christophe Danelon · Ruud Hovius • \\ Horst Vogel
}

Received: 13 March 2006 / Revised: 22 May 2006 / Accepted: 8 June 2006 / Published online: 13 July 2006

(C) EBSA 2006

\begin{abstract}
Nicotinic acetylcholine receptors are heteropentameric ion channels that open upon activation to a single conducting state. The second transmembrane segments of each subunit were identified as channel-forming elements, but their respective contribution in the gating process remains unclear. Moreover, the detailed impact of variations of the membrane potential, such as occurring during an action potential, on the transmembrane domains, is unknown. Residues at the $12^{\prime}$ position, close to the center of each second transmembrane segment, play a key role in channel gating. We examined their functional symmetry by substituting a lysine to that position of each subunit and measuring the electrical activity of single channels. For $12^{\prime}$ lysines in the $\alpha, \gamma$ and $\delta$ subunits rapid transitions between an intermediate and large conductance appeared, which are interpreted as single lysine protonation events. From the kinetics of these transitions we calculated the $\mathrm{p} K_{\mathrm{a}}$ values of respective lysines and showed that they vary differently with membrane hyperpolarization. Respective mutations in $\beta$ or $\epsilon$ subunits gave receptors with openings of either intermediate or large conductance, suggesting extreme $\mathrm{p} K_{\mathrm{a}}$ values in two open state conformations. The results
\end{abstract}

J. Grandl · C. Danelon · R. Hovius · H. Vogel ( $₫)$

Laboratory of Physical Chemistry of Polymers and

Membranes, Ecole Polytechnique Fédérale de Lausanne,

Faculté des Sciences de base, Station 6,

Lausanne 1015, Switzerland

e-mail: horst.vogel@epfl.ch

Present Address:

J. Grandl

Department of Cell Biology, The Scripps Research Institute,

La Jolla, CA 92037, USA demonstrate that these parts of the highly homologous transmembrane domains, as probed by the $12^{\prime}$ lysines, sense unequal microenvironments and are differently affected by physiologically relevant voltage changes. Moreover, observation of various gating events for mutants of $\alpha$ subunits suggests that the open channel pore exists in multiple conformations, which in turn supports the notion of functional asymmetry of the channel.

\begin{abstract}
Abbreviations
nAChR Nicotinic acetylcholine receptor

TM1/TM2 First/second transmembrane segment

GFP Green fluorescent protein
\end{abstract}

\section{Introduction}

The nicotinic acetylcholine receptor (nAChR) is a paradigm of ligand-gated ion channels (Absalom et al. 2004). Muscle-type nAChR are heteropentamers with a subunit stoichiometry $(\alpha 1)_{2}, \beta 1, \delta, \gamma$ (the $\gamma$ subunit being replaced during maturation by the $\epsilon$ subunit) and two binding sites for the natural agonist acetylcholine, located at the interfaces between the $\alpha$ and their adjacent subunits $(\alpha / \delta, \alpha / \gamma$ or $\alpha / \epsilon)$. Upon ligand binding the receptor switches from its closed conformation in apparently one single step to the open state (Maconochie et al. 1995). How the implied structural rearrangements within the asymmetric structure are realized in detail is still unclear (Grutter and Changeux 2001). 
The conformational changes are not necessarily the same for all subunits (Dahan et al. 2004; Grosman and Auerbach 2000) and structural data from electron microscopy from Torpedo californica $\mathrm{nAChR}$ propose that rotational movements of the $\alpha$ subunits promote channel gating (Miyazawa et al. 2003; Unwin 2005).

From various experiments it is known, that in nAChR the highly conserved second transmembrane segments (TM2) of each subunit (Fig. 1) form the membrane spanning part of the channel (Akabas et al. 1994; Hucho et al. 1986). Lysine substitution along the $\delta$-TM2 in mouse nAChR has recently been shown to locally probe the microenvironment of the pore (Cymes et al. 2005), and protonation of the $\epsilon-\mathrm{NH}_{2}$ group reduced channel conductance. Within a channel pore the proton exchange reaction is greatly slowed down so that individual events become measurable compared to bulk conditions, where such processes happen on time scales shorter than the typical time-resolution of patchclamp experiments. The degree of channel blocking reflects, due to repulsion of equal charges, the distance of the lysine to the ion pathway. Gating rate constants directly mirror the protonation kinetics. They can hence be used to calculate local $\mathrm{p} K_{\mathrm{a}}$ values of single lysines in the channel, which might differ from the $\mathrm{p} K_{\mathrm{a}}$ value of lysine in a bulk environment ( 10.2).

Here, we extended this approach to the $12^{\prime}$ positions of TM2 in all subunits. Moreover, we studied the effect of engineered lysines on single-channel properties as a function of membrane potential to sense structural and functional differences between individual subunits.

\section{Materials and methods}

Site-directed mutagenesis

The single-point mutations $\alpha \mathrm{T} 254 \mathrm{~K}, \beta \mathrm{T} 265 \mathrm{~K}, \gamma \mathrm{T} 263 \mathrm{~K}$ $\delta \mathrm{S} 268 \mathrm{~K}$ and $\epsilon \mathrm{T} 263 \mathrm{~K}$ were produced using cDNA coding for subunits of human muscle-type nAChR using the QuikChange Site-Directed Mutagenesis Kit (Stratagene, La Jolla, CA, USA) and their correctness was confirmed by sequencing (MWG, Germany).

\section{Cell preparation}

Human embryonic kidney cells were cultured in DMEM:F-12 medium (Invitrogen, Basel, Switzerland) containing $2.5 \%$ fetal calf serum (Sigma, Buchs, Switzerland) at $37^{\circ} \mathrm{C}$ in a humidified $5 \% \mathrm{CO}_{2}$ atmosphere. Cells were transiently transfected using the Effectene Transfectent Reagent (Qiagen, Hombrechtlikon, Switzerland). A total of $0.2 \mu \mathrm{g}$ cDNA coding for the $\alpha, \beta, \delta$ and $\epsilon$ or $\gamma$ subunits of the human muscle nAChR was mixed with green fluorescent protein (GFP) cDNA in the ratio of $2: 1: 1: 1: 1(\alpha: \beta: \epsilon$ or $\gamma: \delta: \mathrm{GFP})$ or in equal amounts for experiments combining mutated and native $\alpha$ subunits and applied to a $35 \mathrm{~mm}$ culture dish with $2 \mathrm{ml}$ medium. Experiments were performed on the adulttype receptor, for its larger single-channel conductance (59 vs. $39 \mathrm{pS}$ for fetal-type receptors) and therefore its higher signal-to-noise ratio in single-channel measurements (Newland et al. 1995), except for measurements on the $\gamma \mathrm{T} 12$ 'K mutant. Experiments were carried out 13 days after transfection upon transferring cells to a solution containing: $\mathrm{NaCl} 147 \mathrm{mM}$, glucose $12 \mathrm{mM}$, HEPES $10 \mathrm{mM}, \mathrm{KCl} 2 \mathrm{mM}, \mathrm{MgCl}_{2} 1 \mathrm{mM}$ (all Sigma), $\mathrm{pH}$ adjusted to 7.4 with $\mathrm{NaOH}$.

Lysine substitutions in $\alpha$ subunits

To investigate the effects of lysine substitution in each of the two $\alpha$ subunits $\left(\alpha_{\delta}\right.$ or $\left.\alpha_{\epsilon}\right)$, three sets of singlechannel experiments were performed upon transfection of DNA coding for: (i) the adult wild-type receptor $(\alpha, \beta, \epsilon, \delta)$ in the ratio $2: 1: 1: 1$, (ii) only the mutated version of the $\alpha$ subunit $\left(\alpha \mathrm{T} 12^{\prime} \mathrm{K}, \beta, \epsilon, \delta\right)$ in the ratio 2:1:1:1, yielding receptors containing the mutation in both $\alpha$ subunits (further: $2 \times \alpha \mathrm{T} 12^{\prime} \mathrm{K}$ ), (iii) both mutated and wild-type $\mathrm{nAChR}\left(\alpha \mathrm{T} 12^{\prime} \mathrm{K}, \alpha, \beta, \epsilon, \delta\right)$ in
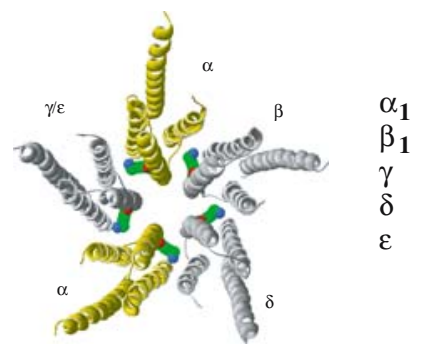

Fig. 1 Channel structure and TM2 alignment of human nAChR. Structure of the nAChR transmembrane segments of Torpedo californica nAChR (Unwin 2005), showing engineered lysines at

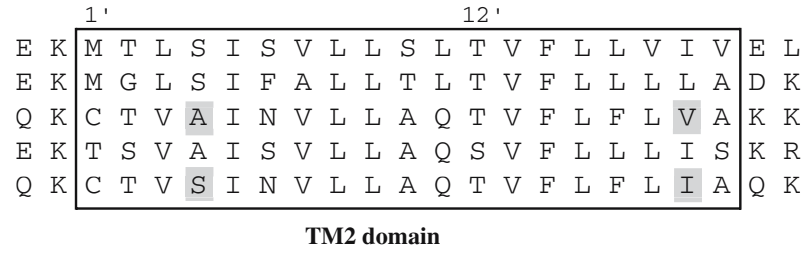

$12^{\prime}$ positions in green (left). Alignment of the TM2 domains of human muscle nAChR subunits (right). Differences within the TM2 (box) residue between subunits $\gamma$ and $\epsilon$ are highlighted 
Fig. 2 Single-channel current traces of $12^{\prime} \mathrm{K}$ nAChR mutants. (a) Predominant gating behaviour when stimulated by $10 \mu \mathrm{M}$ ACh. Downward deflections are channel openings. (b) For $2 \times \alpha \mathrm{T} 12^{\prime} \mathrm{K}$ receptors opening with largely increased open channel noise and stepwise channel closing (marked with arrows) was frequent. (c) For $1 \times \alpha \mathrm{T} 12^{\prime} \mathrm{K}$ receptors two different gating behaviours (marked 1 and 2) with distinct lifetimes ( $\sim 1$ and $\sim 5 \mathrm{~ms})$ that open towards a large conducting state (presumably wild-type conductance) were observed within the same patches a wild-type
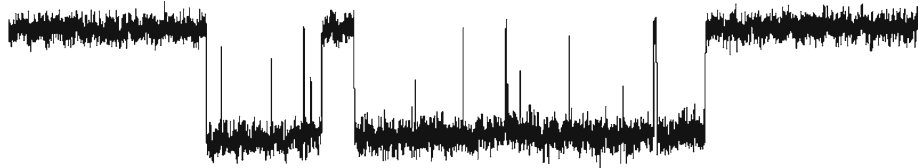

$1 \mathrm{x} \alpha \mathrm{T} 12^{\prime} \mathrm{K}$

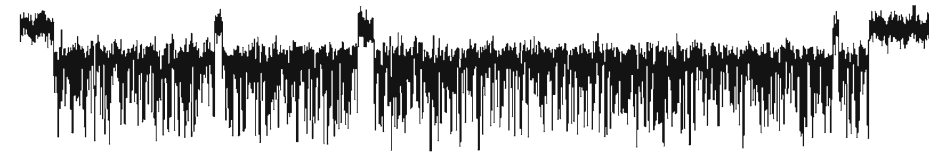

2x $\alpha$ T12'K

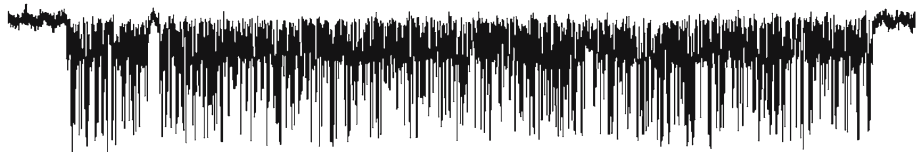

$\beta T 12 ' \mathrm{~K}$

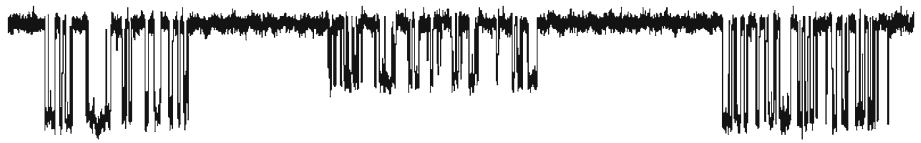

$\gamma \mathrm{T} 12^{\prime} \mathrm{K}$

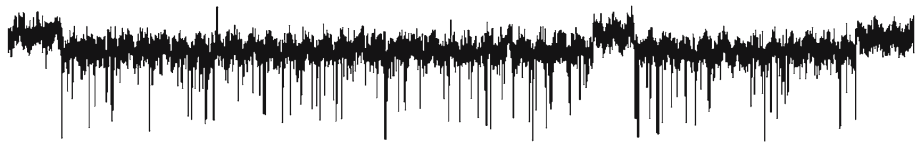

$\delta S 12 ' \mathrm{~K}$
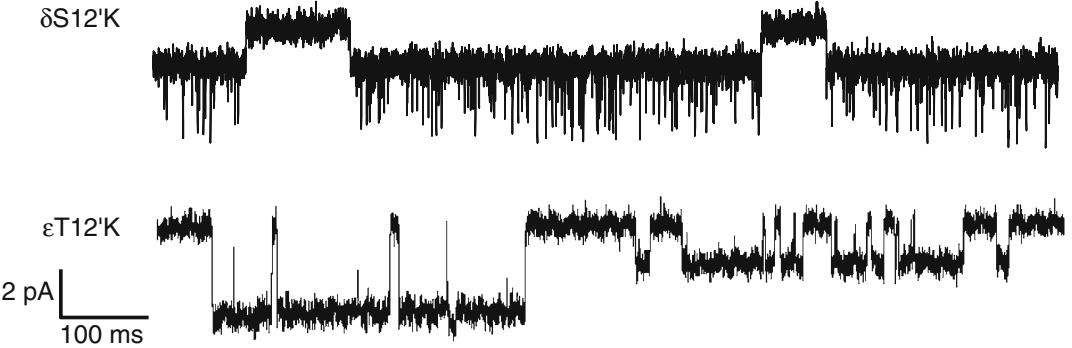

b

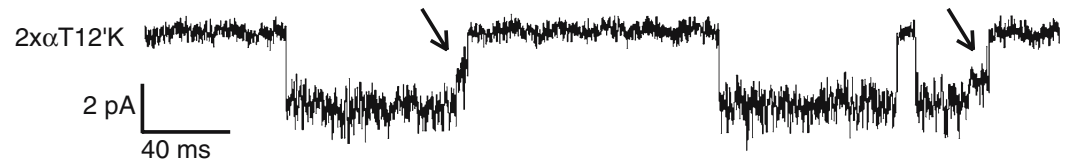

c

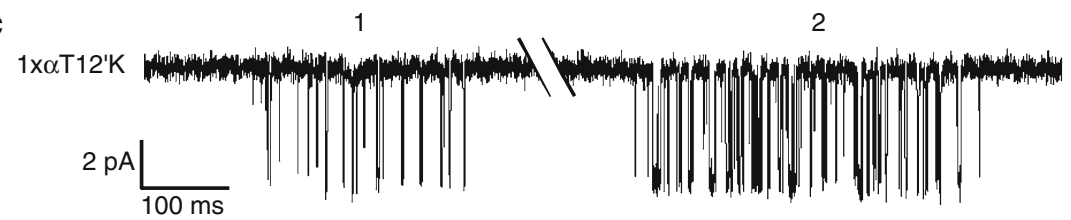

equal amount, resulting in expression of receptors containing either none, one (further $1 \times \alpha \mathrm{T} 12^{\prime} \mathrm{K}$ ) or two $\left(2 \times \alpha \mathrm{T} 12^{\prime} \mathrm{K}\right)$ mutations.

\section{Patch-clamp recordings}

Recordings were performed at $18^{\circ} \mathrm{C}$ in buffer solution (see above) on cells that showed green fluorescence. Borosilicate patch pipettes (GB 150F-8P, Science
Products, Basel, Switzerland) were pulled with a P-87 micropipette puller (Sutter Instruments, Novato, USA) and had a resistance of 5-10 M $\Omega$ when filled with a solution containing $\mathrm{NaCl} 140 \mathrm{mM}$, EGTA $10 \mathrm{mM}$ and HEPES $10 \mathrm{mM}$, pH adjusted to 7.4 with $\mathrm{NaOH}$. Currents were measured with an EPC9 patch-clamp amplifier (HEKA, Lambrecht, Germany).

Single-channel measurements were carried out in the cell-attached configuration with $10 \mu \mathrm{M}$ ACh 

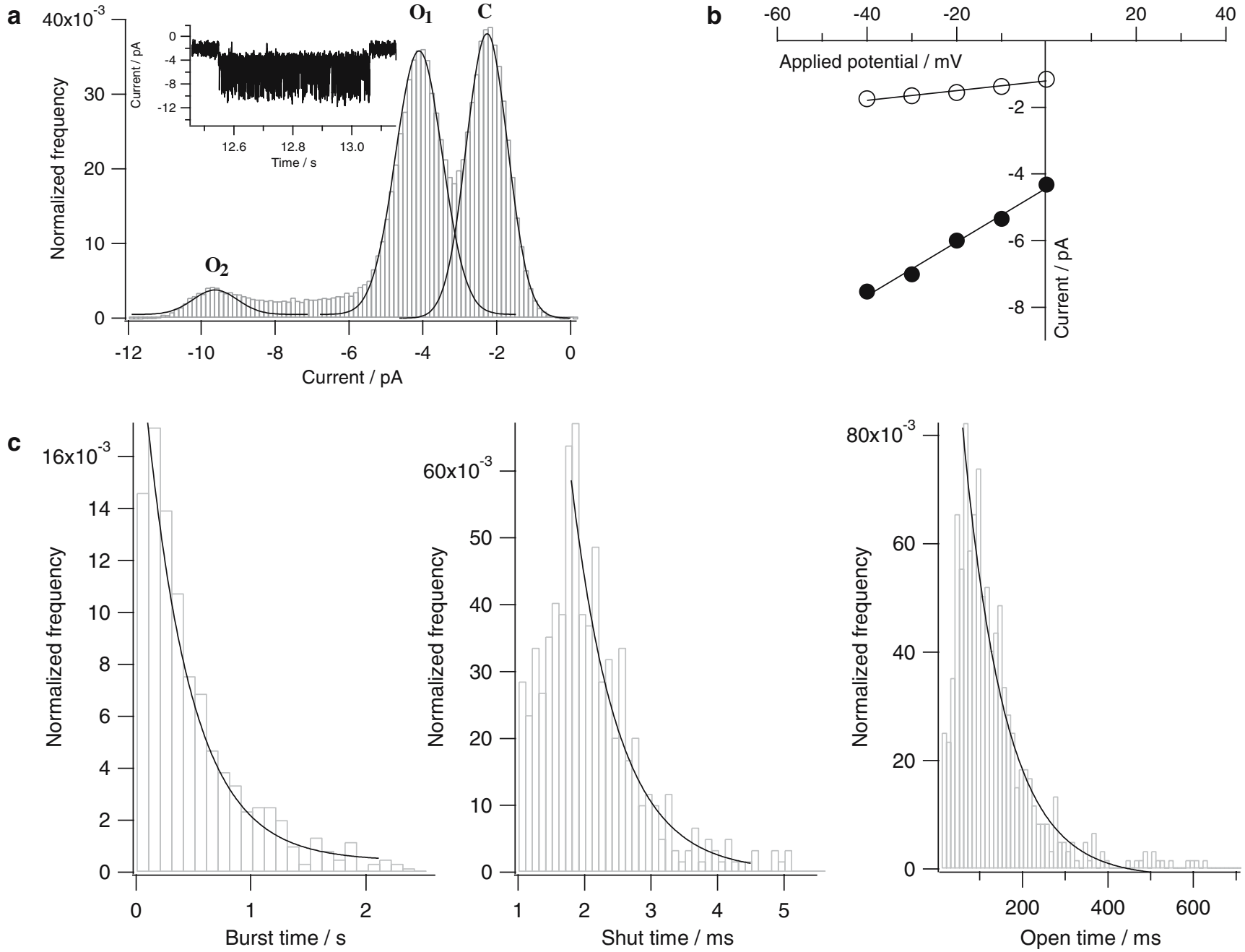

Fig. 3 Analysis of single-channel current traces of the $1 \times \alpha \mathrm{T} 12$ 'K receptor. (a) Amplitude histogram of current trace (inset) fitted with three Gaussian distributions corresponding to the three states $\mathrm{C}, \mathrm{O}_{1}$ and $\mathrm{O}_{2}$. (b) Values of current differences between the closed state $\mathrm{C}$ and the open states $\mathrm{O}_{1}$ (open circle) and $\mathrm{O}_{2}$ (filled circle), deduced from midpoints of Gaussian fits to amplitude histograms as a function of the applied voltage. Lines are linear fits with respective slopes of $14 \pm 2$ and $76 \pm 6 \mathrm{pS}$. (c) Dwell time histograms for burst time $\tau_{\mathrm{b}}$, the small component of the shut time $\tau_{\mathrm{s}}$ and open time $\tau_{\mathrm{o}}$, derived from single-channel current traces. Black lines are single exponential fits to the data with time constants $\tau_{\mathrm{b}}=395 \pm 29, \quad \tau_{\mathrm{s}}=0.7 \pm 0.1$ and $\tau_{\mathrm{o}}=106 \pm 10 \mathrm{~ms}$

the reversal potential in order to obtain the actual value of the transmembrane voltage.

Dwell-time analysis

Segments of current traces showing single-channel activity were selected and idealized using the SKM algorithm of QuB (Qin et al. 1996). Histograms of open-channel time distributions showed a single component and hence could be well fitted with single exponential curves using Igor Pro and their means were calculated (Fig. 3c). Closed-channel time distributions showed two well-separated components. The smaller component had typically a lifetime of $\sim 1 \mathrm{~ms}$ and its 
upper limit (typically $\sim 5 \mathrm{~ms}$, which did not interfere with events of the larger component) was used as a critical time value $t_{\text {crit }}$. In the following, bursts were defined as a series of closely spaced openings into a conducting state that were longer than $20 \mathrm{~ms}$ and preceded and followed by closed intervals longer than $t_{\text {crit }}$.

Rate-kinetics of the fast gating transitions (protonation/deprotonation) were fitted (MIL-algorithm) for each patch experiment separately with correction for missed events, imposing a dead-time of 90-100 $\mu$ s (Qin et al. 1996).

\section{Results and discussion}

Transitions between subconducting and fully conducting states have been observed recently in nAChR containing single lysines in the $\delta$-TM2 domain and were interpreted as protonation-deprotonation events of single $\epsilon-\mathrm{NH}_{2}$ groups in the open channel pore (Cymes et al. 2005). Here, we address the question of symmetry between pore lining domains of the individual subunits by comparing experiments on lysine mutations at identical positions in each subunit.

$12^{\prime}$ Lysine mutation affects gating differently in $\alpha, \gamma$ and $\delta$ than in $\beta$ and $\epsilon$ subunits

Whereas wild-type receptors show openings from a closed state $\mathrm{C}$ to a single open state, receptors with the introduced $12^{\prime}$ lysine in $\alpha, \gamma$ or $\delta$ subunits feature within a burst a large conducting state $\mathrm{O}_{2}$ and an open state $\mathrm{O}_{1}$ of intermediate conductance and rapid transitions between these states, as seen in single-channel patchclamp recordings at pH 7.4 (Fig. 2a). To the contrary, receptors $\beta \mathrm{T} 12^{\prime} \mathrm{K}$ and $\epsilon \mathrm{T} 12^{\prime} \mathrm{K}$ opened either to a state of intermediate $\mathrm{O}_{1}$ or large $\mathrm{O}_{2}$ conductance and direct transitions between $\mathrm{O}_{1}$ and $\mathrm{O}_{2}$ have not been observed (Fig. 2a).

Different assemblies and gating behaviors for alpha mutated receptors

Co-transfection of both alpha wild-type and mutant $\alpha \mathrm{T} 12^{\prime} \mathrm{K}$ cDNA yields stochastic assembly of wild-type, $2 \times \alpha \mathrm{T} 12^{\prime} \mathrm{K}$ and $1 \times \alpha \mathrm{T} 12^{\prime} \mathrm{K}$ hybrid receptors (see methods), with the latter carrying the mutations in either $\alpha_{\delta}$ or $\alpha_{\epsilon}$ subunit. To identify openings specific to $1 \times \alpha \mathrm{T} 12$ 'K hybrids we removed gating events similar to those observed for measurements with wild-type or $2 \times \alpha \mathrm{T} 12$ 'K receptors. Amongst the remaining openings we observed predominantly openings with intermediate conductance (Fig. 2a) and more rarely openings from the closed state towards a large conducting state (presumably identical to $\mathrm{O}_{2}$ ), with two distinct lifetimes (Fig. 2b). These two gating types might reflect the two possible populations of $1 \times \alpha \mathrm{T} 12^{\prime} \mathrm{K}$ receptors containing the lysine in either $\alpha_{\delta}$ or $\alpha_{\epsilon}$ subunit.

$2 \times \alpha \mathrm{T} 12^{\prime} \mathrm{K}$ receptors showed in addition within bursts, next to $\mathrm{O}_{1} \rightleftarrows \mathrm{O}_{2}$ transitions, frequent fast transitions from the subconductance state $\mathrm{O}_{1}$ to a closed or low conductance state. We assign this transition to the protonation of the second $\epsilon-\mathrm{NH}_{2}$ group, since its voltage dependence is similar to that of the first protonation, as shown in detail below.

In addition to the mentioned activities (Fig. 2a) alternative gating features were occasionally $(\sim 2 \%$ of all bursts) observed for the $2 \times \alpha \mathrm{T} 12^{\prime} \mathrm{K}$ containing receptors (Fig. $2 \mathrm{c}$ ). $2 \times \alpha \mathrm{T} 12^{\prime} \mathrm{K}$ receptors open to a large conductance state with largely increased open channel noise and channel closing through intermediate conducting states. These channel activities were observed sequentially (not simultaneously) to the ones shown in Fig. $2 \mathrm{a}$ in the same patches, indicating that this gating does not stem from multiple channels with different subunit assemblies, (mis-)foldings or conformations, but rather from one single channel. This demonstrates further complexity of open channel conformations and their gating pathways.

All mutant receptors gate between two open states

The existence of only two open states is most obvious for $\beta \mathrm{T} 12^{\prime} \mathrm{K}$ and $\epsilon \mathrm{T} 12^{\prime} \mathrm{K}$ receptors due to their long lifetimes. Since receptors containing lysines engineered in $\alpha, \gamma$ and $\delta$ subunits showed gating with much shorter lifetimes, we analyzed power density spectra of current fluctuations for the exemplary $\delta \mathrm{S} 12^{\prime} \mathrm{K}$ mutant. We obtained single Lorentzian curves (not shown), supporting that the rapid current fluctuations are also well described by a simple two-state Markov process (Sigworth 1985). Therefore, we interpret these transitions as protonation events.

Channel currents are blocked dissimilarly by protonation of different $12^{\prime}$ lysines

Conductance values $g_{1}$ and $g_{2}$ of the states $\mathrm{O}_{1}$ and $\mathrm{O}_{2}$, obtained as shown in Fig. 3, are summarized for all receptors in Table 1. We calculated the extent of channel block

$\left(g_{2}-g_{1}\right) / g_{2}$

which is thought to reflect the proximity of the lysine to the ion pathway (Cymes et al. 2005). The value of the $\delta$ 
subunit is significantly lower $(56 \pm 14 \%)$ than those of $\alpha$ and $\gamma$ subunit $(82 \pm 13$ and $82 \pm 17 \%)$. The conductance value corresponding to the second protonation event is below the resolution limit and indistinguishable from zero and might reflect a nearly completely blocked ion pathway.

Current differences between states $\mathrm{O}_{1}$ and $\mathrm{O}_{2}$ are relatively small for receptors $\beta \mathrm{T} 12^{\prime} \mathrm{K}$ and $\epsilon \mathrm{T} 12^{\prime} \mathrm{K}$ compared to the other receptors, suggesting little channel blocking ( $40 \pm 11$ and $27 \pm 8 \%$, respectively). However, as discussed later, we cannot be sure that lysine protonation is the origin. For this reason we limited further analysis concerning these two mutants to the determination of gating rate constants for $\mathrm{O}_{2}$ at different membrane potentials (Fig. 4).

Analysis of gating kinetics reveals voltage dependence of $\mathrm{p} K_{\mathrm{a}}$ values

In the following we focus on receptors $1 \times \alpha \mathrm{T} 12^{\prime} \mathrm{K}$, $2 \times \alpha \mathrm{T} 12^{\prime} \mathrm{K}, \gamma \mathrm{T} 12^{\prime} \mathrm{K}$ and $\delta \mathrm{T} 12^{\prime} \mathrm{K}$, with emphasis on their fast gating transitions (as shown in Fig. 2a), which we associate with $12^{\prime} \mathrm{K}$ lysine protonation. Shut times $\tau_{\mathrm{s}}$, open times $\tau_{\mathrm{o}} \mathrm{C} \rightleftarrows \mathrm{O}_{1}+\mathrm{O}_{2}$ and burst times were not voltage dependent within experimental error for receptors $1 \times \alpha \mathrm{T} 12^{\prime} \mathrm{K}, \gamma \mathrm{T} 12^{\prime} \mathrm{K}$ and $\delta \mathrm{T} 12^{\prime} \mathrm{K}$. We observed that burst times of $\delta \mathrm{S} 12^{\prime} \mathrm{K}$ receptors were about two-fold larger $(757 \pm 159 \mathrm{~ms})$ than that of $1 \times \alpha \mathrm{T} 12^{\prime} \mathrm{K}$ $(395 \pm 29 \mathrm{~ms})$ or $\gamma \mathrm{T} 12^{\prime} \mathrm{K}(414 \pm 58 \mathrm{~ms})$ receptors, whereas respective apparent equilibrium constants did not vary significantly (Table 1 ). Presently, we cannot interpret this feature in concrete structural or functional terms. However, together with the significantly different extent of channel block it is evident that the lysine engineered in the $\delta$ subunit is not located identically to lysines in $\alpha$ and $\gamma$ subunits with respect to the ion pathway and that the gating transmission of the $\delta$ subunit differs to that from other subunits.

Assuming a first-order proton binding reaction to lysine side chains, the protonation rate $k_{21}\left(\mathrm{~s}^{-1}\right)$ is defined as the closing rate from state $\mathrm{O}_{2}$ to $\mathrm{O}_{1}$, while the deprotonation rate $k_{12}\left(\mathrm{~s}^{-1}\right)$ is defined as the opening rate from $\mathrm{O}_{1}$ to $\mathrm{O}_{2}$. The actual protonation rate constant (in $\mathrm{M}^{-1} \mathrm{~s}^{-1}$ ) is given by $k_{21} /\left[\mathrm{H}^{+}\right]$. Figure 5 shows that $k_{12}$ and $k_{21}$ (and additionally the rate constants of channel gating $\mathrm{C} \rightleftarrows \mathrm{O}_{1}$ for $\left.2 \times \alpha \mathrm{T} 12^{\prime} \mathrm{K}\right)$ are voltage dependent. Whereas $k_{21}$ was larger than $k_{12}$ at all examined voltages, the latter increased more strongly with membrane hyperpolarization (Fig. 5). Extrapolated values at zero voltage are reported in Table 2 . We calculated $\mathrm{p} K_{\mathrm{a}}$ values of individual lysines at $\mathrm{pH} 7.4$ as

$\mathrm{p} K_{\mathrm{a}}=-\log \left(k_{12}^{\prime} \times\left[\mathrm{H}^{+}\right] / k_{21}\right)$

(Fig. 6 and Table 2).

Both, the $\mathrm{p} K_{\mathrm{a}}$ value and extent of channel block found for the $\delta \mathrm{S} 12^{\prime} \mathrm{K}$ receptor are in good agreement with similar measurements on the mouse nAChR (Cymes et al. 2005), which shows that the high homology between these receptors on the level of the primary sequence $(>98 \%)$ seems also to extend to the structural level. Additionally, we found that all $\mathrm{p} K_{\mathrm{a}}$ values, except for $\gamma \mathrm{T} 12^{\prime} \mathrm{K}$, decrease with membrane hyperpolarization, which is mainly due to the voltage dependence of the deprotonation rate. This might result from reorientation of these residues towards a more hydrophobic environment with hyperpolarization or the increased electric force on the proton.

Extrapolation of the data shows, that $\mathrm{p} K_{\mathrm{a}}$ values for protonation of one $12^{\prime}$ lysine would be similar at zero voltage ( 8.6). This might reflect that at zero voltage the lysines have virtually identical microenvironments, which diverge with membrane hyperpolarization. We speculate that the observed voltage dependence of $\mathrm{p} K_{\mathrm{a}}$ values (which ranks $\gamma \mathrm{T} 12^{\prime} \mathrm{K}<\delta \mathrm{S} 12^{\prime} \mathrm{K} \sim 1 \times \alpha \mathrm{T} 12^{\prime} \mathrm{K}$ ) might reflect the degree of displacement of the respective $12^{\prime}$ residue with membrane polarization. Protonation of the second lysine in the $2 \times \alpha \mathrm{T} 12^{\prime} \mathrm{K}$ receptor seems to be less favorable $(\Delta \mathrm{G} \approx 3.9 \mathrm{~kJ} / \mathrm{mol}$

Table 1 Single channel conductance and dwell time analysis of $12^{\prime} \mathrm{K}$ receptors

\begin{tabular}{|c|c|c|c|c|c|c|c|c|}
\hline Receptor & $g_{1}(\mathrm{pS})$ & $g_{2}(\mathrm{pS})$ & $\begin{array}{l}\text { Channel } \\
\text { block (\%) }\end{array}$ & $\begin{array}{l}\text { Burst } \\
\text { length }(\mathrm{ms})\end{array}$ & $\begin{array}{l}\text { Shut time } \\
\tau_{\mathrm{s}}(\mathrm{ms})\end{array}$ & $\begin{array}{l}\text { Open time } \\
\tau_{\mathrm{o}}(\mathrm{ms})\end{array}$ & $\begin{array}{l}\text { Apparent } \\
\text { equilibrium } \\
\text { constant } \Theta\end{array}$ & No. \\
\hline $1 \times \alpha \mathrm{T} 12^{\prime} \mathrm{K}$ & $14 \pm 2$ & $76 \pm 6$ & $82 \pm 17$ & $395 \pm 29$ & $0.7 \pm 0.1$ & $106 \pm 10$ & $151 \pm 33$ & 4 \\
\hline $2 \times \alpha \mathrm{T} 12^{\prime} \mathrm{K}$ & $26 \pm 2$ & $76 \pm 3$ & $66 \pm 9$ & - & - & - & - & 2 \\
\hline$\beta \mathrm{T} 12^{\prime} \mathrm{K}$ & $42 \pm 5$ & $70 \pm 2$ & $40 \pm 11$ & - & - & - & - & 3 \\
\hline$\gamma \mathrm{S} 12^{\prime} \mathrm{K}$ & $11 \pm 2$ & $60 \pm 3$ & $82 \pm 13$ & $414 \pm 58$ & $1.0 \pm 0.2$ & $111 \pm 25$ & $111 \pm 59$ & 3 \\
\hline$\delta \mathrm{T} 12^{\prime} \mathrm{K}$ & $25 \pm 2$ & $57 \pm 4$ & $56 \pm 14$ & $757 \pm 159$ & $1.1 \pm 0.2$ & $205 \pm 27$ & $189 \pm 69$ & 4 \\
\hline$\epsilon \mathrm{T} 12^{\prime} \mathrm{K}$ & $55 \pm 2$ & $75 \pm 3$ & $27 \pm 8$ & - & - & - & - & 3 \\
\hline
\end{tabular}

Single channel conductances $\mathrm{g}_{1}$ and $\mathrm{g}_{2}$, extent of channel block $\left(g_{2}-g_{1}\right) / g_{2}$ in percent, burst length, shut time $\tau_{\mathrm{s}}$, open time $\tau_{\mathrm{o}}$, apparent gating equilibrium constant $\Theta=\tau_{\mathrm{o}} / \tau_{\mathrm{s}}$ and number of patches evaluated for the gating transition between the closed state $\mathrm{C}$ and the open states $\mathrm{O}_{1}$ and $\mathrm{O}_{2}$ 
Fig. 4 Rates for channel closing (filled square) and channel opening (open square) for the transition $\mathrm{C} \rightleftarrows \mathrm{O}_{2}$ as a function of voltage for $\beta \mathrm{T} 12^{\prime} \mathrm{K}(\mathbf{a})$ and $\epsilon \mathrm{T} 12^{\prime} \mathrm{K}(\mathbf{b})$ receptors. Fitting (lines) the data to $k(V)=k_{V=0}$ $\exp (-(z \delta \mathrm{F} / \mathrm{RT}) V)$ gave for $\beta \mathrm{T} 12^{\prime} \mathrm{K}: k_{12, V=0}=138 \pm 20 \mathrm{~s}^{-}$ ${ }^{1}, \mathrm{z} \delta=-0.01 \pm 0.03$,

$k_{21, V=0}=3,779 \pm 307 \mathrm{~s}^{-1}$,

$\mathrm{z} \delta=-0.39 \pm 0.02$ and $\epsilon \mathrm{T} 12^{\prime} \mathrm{K}:$

$k_{12, V=0}=84 \pm 39 \mathrm{~s}^{-1}$,

$z \delta=0.04 \pm 0.10$,

$k_{21, V=0}=8,913 \pm 3,740 \mathrm{~s}^{-1}$

$z \delta=-0.40 \pm 0.10$
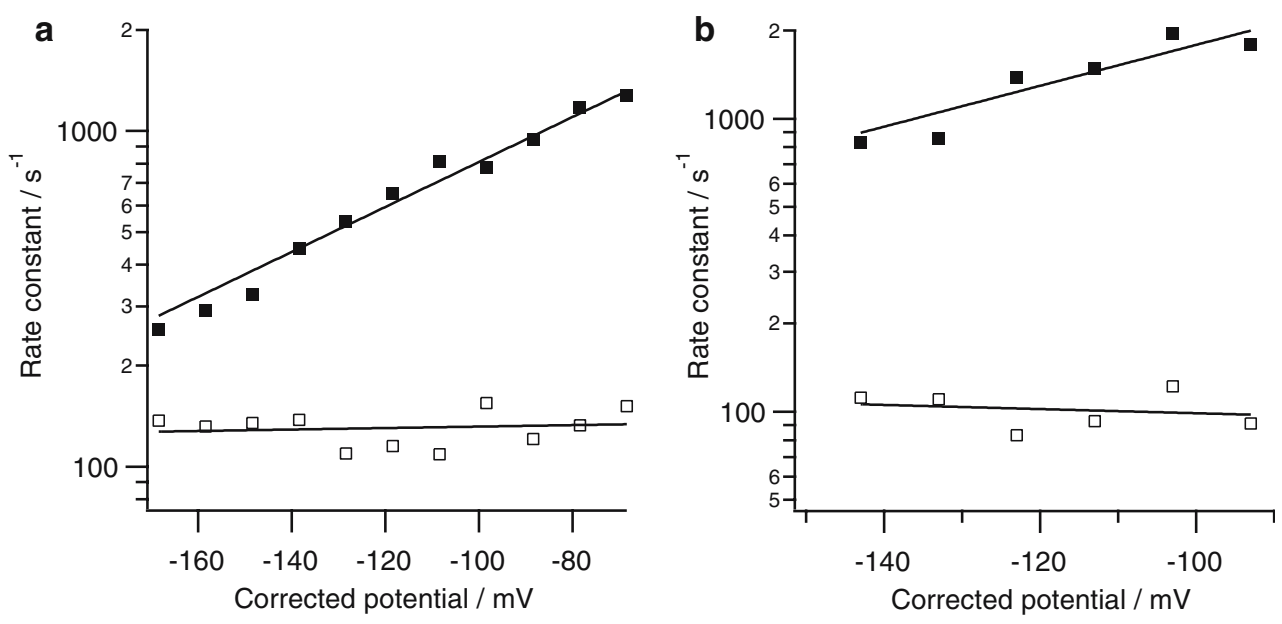

at $0 \mathrm{~V})$ as compared to protonation of the first lysine, as can be seen from the lower $\mathrm{p} K_{\mathrm{a}, V=0}$ value $(\sim 7.7)$. This is consistent, considering that the first protonated lysine hinders the second protonation event due to electrostatic repulsion. The observed voltage dependence demonstrates also that the precision of $\mathrm{p} K_{\mathrm{a}}$ values determined by this approach is at least ten-fold lower as estimated before (Cymes et al. 2005), since it is dominated by the error of the exact membrane potential measurement.
Fig. 5 Rates of the first $\left[\mathrm{k}_{12}\right.$ (open square) $/ k_{21}$ (filled square $] \mathrm{O}_{1} \rightleftarrows \mathrm{O}_{2}$ and the second $\left[\mathrm{k}_{12}\right.$ (open circle)/ $k_{21}$ (filled circle)] $\mathrm{C} \rightleftarrows \mathrm{O}_{1}$ channel blocking (only receptor $\left.2 \times \alpha \mathrm{T} 12^{\prime} \mathrm{K}\right)$ as a function of the corrected potential for receptors $1 \times \alpha \mathrm{T} 12^{\prime} \mathrm{K}(\mathbf{a})$, $2 \times \alpha \mathrm{T} 12^{\prime} \mathrm{K}(\mathbf{b}), \gamma \mathrm{T} 12^{\prime} \mathrm{K}(\mathbf{c})$ and $\delta \mathrm{T} 12^{\prime} \mathrm{K}(\mathbf{d})$. Parameters of fits (solid lines) are summarized in Table 2
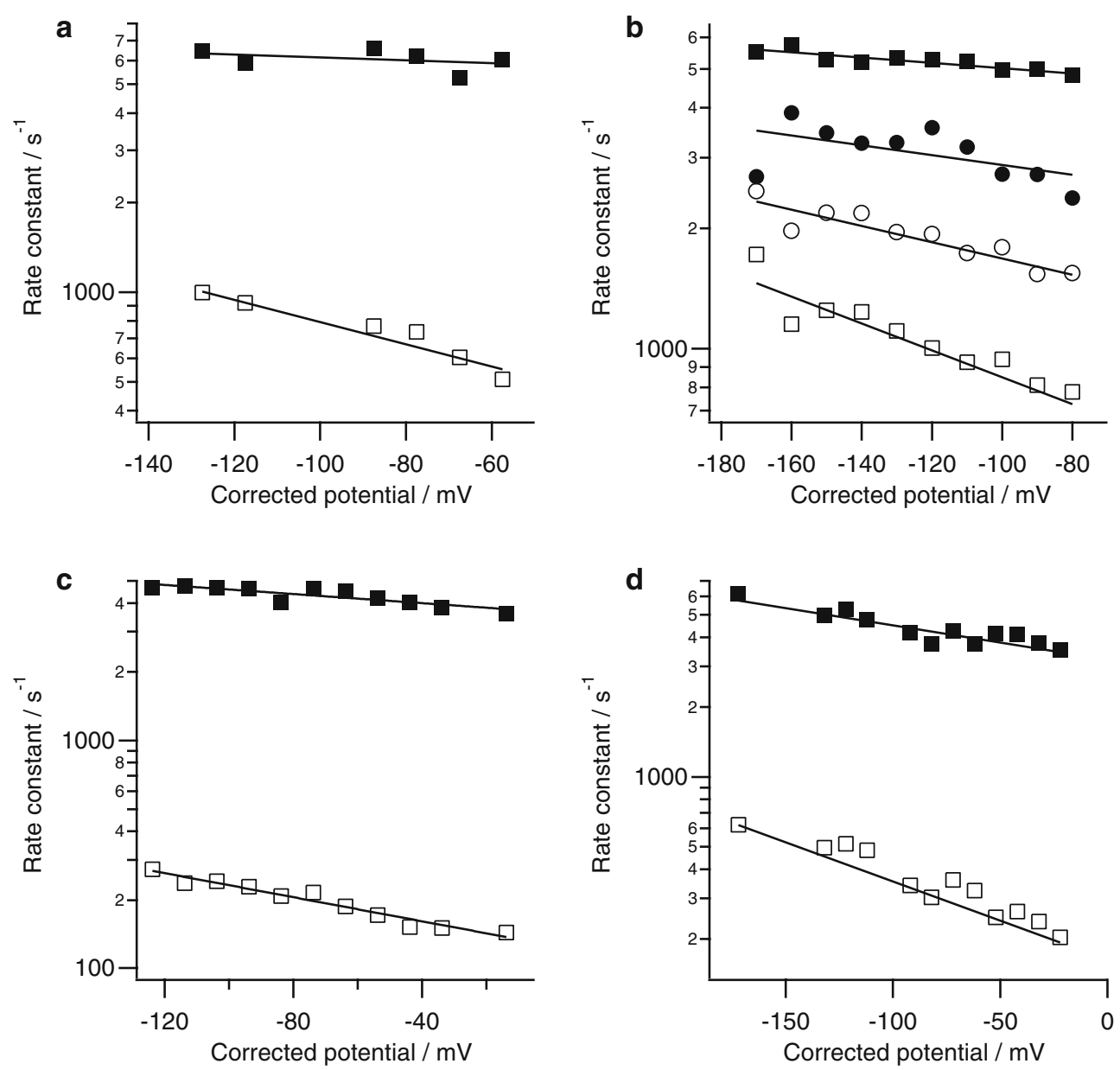

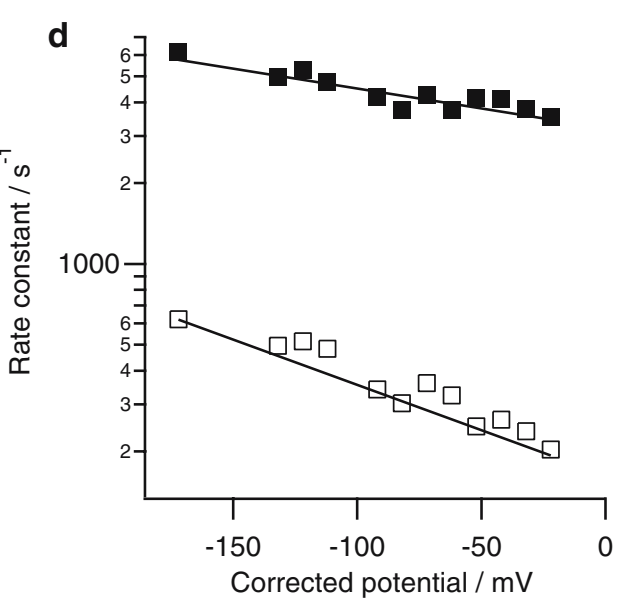


Table 2 Voltage dependence of (de-)protonation rates and $\mathrm{p} K_{\mathrm{a}}$ values

\begin{tabular}{|c|c|c|c|c|c|c|}
\hline \multirow[t]{2}{*}{ Receptor } & \multicolumn{2}{|c|}{ Deprotonation } & \multicolumn{2}{|l|}{ Protonation } & \multicolumn{2}{|l|}{$\mathrm{p} K_{\mathrm{a}}$} \\
\hline & $k_{12, V=0} \mathrm{~s}^{-1}$ & $z \delta$ & $k_{21, V=0} \mathrm{~s}^{-1}$ & $z \delta$ & $\mathrm{p} K_{\mathrm{a}, V=0}$ & $z^{\prime} \delta^{\prime}$ \\
\hline $1 \times \alpha \mathrm{T} 12^{\prime} \mathrm{K}$ & $256 \pm 36$ & $0.33 \pm 0.04$ & $2,311 \pm 381$ & $0.09 \pm 0.05$ & $8.6 \pm 0.1$ & $0.08 \pm 0.02$ \\
\hline$\gamma \mathrm{S} 12^{\prime} \mathrm{K}$ & $127 \pm 5$ & $0.15 \pm 0.01$ & $3,644 \pm 139$ & $0.06 \pm 0.01$ & $8.7 \pm 0.1$ & $-0.01 \pm 0.01$ \\
\hline$\delta \mathrm{T} 12^{\prime} \mathrm{K}$ & $195 \pm 14$ & $0.18 \pm 0.02$ & $3245 \pm 148$ & $0.09 \pm 0.01$ & $8.7 \pm 0.1$ & $0.06 \pm 0.01$ \\
\hline $2 \times \alpha \mathrm{T} 12^{\prime} \mathrm{K}_{(1)}$ & $406 \pm 71$ & $0.19 \pm 0.03$ & $4,332 \pm 155$ & $0.04 \pm 0.01$ & $8.4 \pm 0.1$ & $0.06 \pm 0.01$ \\
\hline $2 \times \alpha \mathrm{T} 12^{\prime} \mathrm{K}_{(2)}$ & $1,076 \pm 109$ & $0.12 \pm 0.02$ & $2,242 \pm 440$ & $0.07 \pm 0.04$ & $7.7 \pm 0.1$ & $0.02 \pm 0.02$ \\
\hline
\end{tabular}

Fit values to opening and closing rates and $\mathrm{p} K_{\mathrm{a}}$ values as a function of voltage $\mathrm{V}$, according to $k(V)=k_{V=0} \exp (-(z \delta \mathrm{F} / \mathrm{RT}) V)$ and $\mathrm{p} K_{\mathrm{a}}(V)=\mathrm{p} K_{\mathrm{a}, V=0}+\left(z^{\prime} \delta^{\prime} \mathrm{F} / \mathrm{RT}\right) V$ (Auerbach et al. 1996) (Figs. 5, 6), where $z$ is the amount of charge moving across the fraction $\delta$ of the electric field, $F$ the Faraday constant, $R$ the gas constant and $T$ the temperature. For the receptor $2 \times \alpha \mathrm{T} 12^{\prime} \mathrm{K}$ the first $\left(\mathrm{O}_{2} \rightleftarrows \mathrm{O}_{1}\right)$ and the second ( $\mathrm{O}_{1} \rightleftarrows \mathrm{C}$ ) protonation events are indexed (1) and (2), respectively

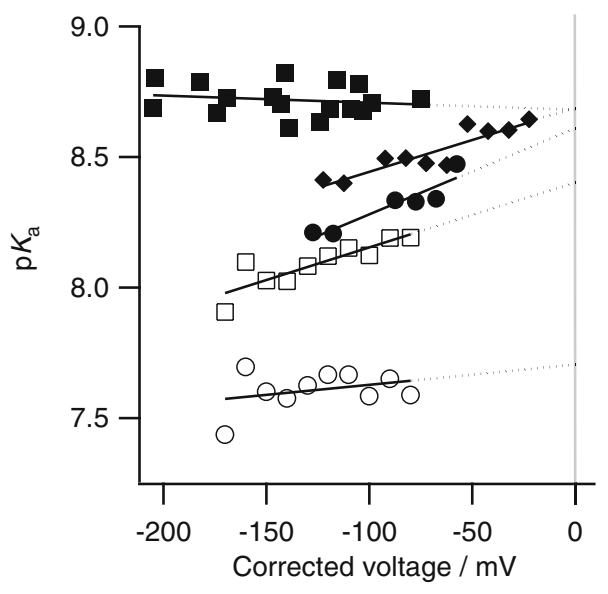

Fig. $6 \mathrm{p} K_{\mathrm{a}}$ values as a function of voltage. Calculated $\mathrm{p} K_{\mathrm{a}}$ values for introduced $12^{\prime}$ lysines as function of voltage for $2 \times \alpha \mathrm{T} 12^{\prime} \mathrm{K}$ (first blocking: open square, second blocking: open circle), $1 \times \alpha \mathrm{T} 12^{\prime} \mathrm{K}$ (filled circle), $\gamma \mathrm{T} 12^{\prime} \mathrm{K}$ (filled square) and $\delta \mathrm{S} 12^{\prime} \mathrm{K}$ (filled diamond) receptors. Solid lines are linear fits to the data, dashed lines extrapolations thereof, and the grey line indicates zero voltage. Fit parameters are summarized in Table 2

The different gating of receptors with $\beta \mathrm{T} 12^{\prime} \mathrm{K}$ or $\epsilon \mathrm{T} 12^{\prime} \mathrm{K}$ might have another origin

Similar gating behaviour and single-channel conductances to that of the $\beta \mathrm{T} 12^{\prime} \mathrm{K}$ receptor have been already observed after transfection of Xenopus oocytes with cDNA for all but $\beta$ subunits (Camacho et al. 1993). We can therefore not exclude that at least in the case of the $\beta \mathrm{T} 12^{\prime} \mathrm{K}$ mutation efficient assembly with wild-type stoichiometry might be compromised. However, assuming a correctly assembled receptor, the lack of fast channel blocking ( $\sim 1 \mathrm{~ms})$ would indicate that lifetimes of protonated and deprotonated states are much larger than the respective burst lifetimes ( $100 \mathrm{~ms})$, which could be explained by extreme $\mathrm{p} K_{\mathrm{a}}$ values of the respective lysines in at least two largely different environments. From the above values we can estimate (see Eq. 2) that $\mathrm{p} K_{\mathrm{a}}$ values of the lysines in the open states $\mathrm{O}_{1}$ and $\mathrm{O}_{2}$ would be superior to $\sim 9.4$ and inferior to $\sim 5.4$, respectively, in order to explain that no fast current fluctuations are observed.

Differences between highly homologous subunits $\gamma$ and $\epsilon$

The difference in gating behavior of $\gamma \mathrm{T} 12^{\prime} \mathrm{K}$ and $\epsilon \mathrm{T} 12^{\prime} \mathrm{K}$ receptors is unexpected, since their respective TM2 regions differ only in positions $4^{\prime}$ and $18^{\prime}$ (Fig. 1). Their overall primary sequence homology is $>90 \%$, which shows that this approach is not limited to the structural level, but is rather sensitive to the functional contributions of subunits. The structure of Torpedo californica nAChR (Fig. 1) suggests that the introduced $12^{\prime}$ lysines in the muscle nAChR do not point directly towards the channel, but rather feature close proximity to TM1 (position 12') and TM2 (position 14') of the adjacent subunit. However molecular dynamics simulations show the $12^{\prime}$ residue to be much closer to the central pore axis and the conformational flexibility of lysines opens multiple structural possibilities, making it difficult to determine specific interactions (Kim et al. 2004).

\section{Conclusion}

Lysines $12^{\prime}$ of TM2 of the different subunits are probing different microenvironments as reflected by their different gating behaviours, their respective kinetic parameters and the voltage dependence of their $\mathrm{p} K_{\mathrm{a}}$ values. The latter is different for each subunit, and reflects and quantifies the structural distortions such as they might occur during channel gating.

Acknowledgments We thank Karen Martinez for critical manuscript reading and Pierre-Jean Corringer and David Beeson for providing cDNA of wild-type nAChR subunits. This work was supported by the Swiss National Science Foundation 
(31-57023.99 and 31-00A0-102062/1) and the European Commission via contract LSHG-CT-2004-504601 (E-MeP).

\section{References}

Absalom NL, Lewis TM, Schofield PR (2004) Mechanisms of channel gating of the ligand-gated ion channel superfamily inferred from protein structure. Exp Physiol 89:145-153

Akabas MH, Kaufmann C, Archdeacon P, Karlin A (1994) Identification of acetylcholine receptor channel-lining residues in the entire M2 segment of the alpha subunit. Neuron 13:919-927

Auerbach A, Sigurdson W, Chen J, Akk G (1996) Voltage dependence of mouse acetylcholine receptor gating: different charge movements in di-, mono- and unliganded receptors. J Physiol 494(Pt 1):155-170

Camacho P, Liu Y, Mandel G, Brehm P (1993) The epsilon subunit confers fast channel gating on multiple classes of acetylcholine receptors. J Neurosci 13:605-613

Cymes GD, Ni Y, Grosman C (2005) Probing ion-channel pores one proton at a time. Nature 438:975-980

Dahan DS, Dibas MI, Petersson EJ, Auyeung VC, Chanda B, Bezanilla F, Dougherty DA, Lester HA (2004) A fluorophore attached to nicotinic acetylcholine receptor beta M2 detects productive binding of agonist to the alpha delta site. Proc Natl Acad Sci USA 101:10195-10200

Grosman C, Auerbach A (2000) Asymmetric and independent contribution of the second transmembrane segment $12^{\prime}$ residues to diliganded gating of acetylcholine receptor channels: a single-channel study with choline as the agonist. J Gen Physiol 115:637-651
Grutter T, Changeux JP (2001) Nicotinic receptors in wonderland. Trends Biochem Sci 26:459-463

Hucho F, Oberthur W, Lottspeich F (1986) The ion channel of the nicotinic acetylcholine receptor is formed by the homologous helices M II of the receptor subunits. FEBS Lett 205:137-142

Maconochie DJ, Fletcher GH, Steinbach JH (1995) The conductance of the muscle nicotinic receptor channel changes rapidly upon gating. Biophys J 68:483-490

Miyazawa A, Fujiyoshi Y, Unwin N (2003) Structure and gating mechanism of the acetylcholine receptor pore. Nature 423:949-955

Kim S, Aaron K. Chamberlain, James U. Bowie (2004). A model of the closed form of the nicotinic acetylcholine receptor $\mathrm{m} 2$ channel pore. Biophys J 87:792-799

Newland CF, Beeson D, Vincent A, Newsom-Davis J (1995) Functional and non-functional isoforms of the human muscle acetylcholine receptor. J Physiol 489(Pt 3):767-778

Qin F, Auerbach A, Sachs F (1996) Estimating single-channel kinetic parameters from idealized patch-clamp data containing missed events. Biophys J 70:264-280

Sigworth FJ (1985) Open channel noise I. Noise in acetylcholine receptor currents suggests conformational fluctuations. Biophys J 47:709-720

Unwin N (2005) Refined structure of the nicotinic acetylcholine receptor at 4A resolution. J Mol Biol 346:967-989 